\title{
Profile of parasuicide cases attending Brits Hospital, North West Province, South Africa: a 5- year chart review
}

Tombo Bongongo ( $\sim$ bongongotombo@gmail.com )

Sefako Makgatho Health Sciences University

K Nkuinika

Brits

DK Nzaumvila

Sefako Makgatho Health Sciences University

A Adfolalu

Sefako Makgatho Health Sciences University

H Okonta

Sefako Makgatho Health Sciences University

I Govender

University of Pretoria

CNS Saidiya

Sefako Makgatho Health Sciences University

Research article

Keywords: Profile of 447 parasuicide cases; Brits Hospital; South Africa; 5-year chart review: 2013-2018.

Posted Date: August 19th, 2020

DOI: https://doi.org/10.21203/rs.3.rs-56437/v1

License: (c) (1) This work is licensed under a Creative Commons Attribution 4.0 International License.

Read Full License 


\section{Abstract}

Background Parasuicide is referred to as an apparent attempt at suicide without the actual intention of killing oneself. Its prevalence continues to increase in the Brits/Madibeng community. The current study aimed to determine the profile of parasuicide cases attending Brits Hospital, North West Province of South Africa, from 2013 to 2018.

Methods The basis of this article is a 5-year retrospective chart review of parasuicide cases attending Brits Hospital from 01 January 2013 to 01 January 2018. Brits Hospital is a 200-bed hospital serving people in the Brits/Madibeng community.

Results Of the 477 parasuicide cases, the mean age was 26.6, the median 24 years with minimum and maximum ages respectively of 12 and 64 years. The majority $(257 ; 53.88 \%)$ were between 16 and 25 years of age and comprised females $(297 ; 62.26 \%)$, African $(391 ; 82.84 \%)$, single people $(352 ; 73.79 \%)$, unemployed $(304 ; 63.7 \%)$ and those who had completed the secondary level of education, $300(62.9 \%)$. The highest number of cases (85, 86 and 87) were registered during the odd years (2013, 2015 and 2017), while the lowest number $(68,75$ and 76) during even years, i.e. 2014, 2016 and 2017. The summer months of January and February registered more cases, respectively $65(13.63 \%)$ and $64(13.42 \%)$. The majority of cases $(430 ; 90.15 \%)$ represented a first attempt at suicide. Overdose of medication (300; $62.82 \%$ ) was the most common method used and a relationship problem the main reason that led to the attempt. Most cases $(409 ; 85.74 \%)$ did not have any medical or psychiatric symptoms prior to the parasuicide crisis.

Conclusion This study painted a clear picture of parasuicidal cases attended at Brits Hospital, being younger between 16-25, mainly female, single, unemployed, the method used for parasuicide mostly overdose of medication and done mainly in Januaries and Februaries. Furthermore, reason for the parasuicidal attempt were relationship problem as well as unemployment. In most of the cases, it was the first attempt.

\section{Background}

Defined as a suicide attempt and deliberate self-harm inflicted with no intent to die, parasuicide ${ }^{1}$ is a public health concern in some parts of the world. ${ }^{2}$ The emergency unit at Brits Hospital receives many of these cases. For the past five years on average 10-15 parasuicide cases per month were reported in some months of the year. The frequency of the phenomenon explains the initiation of the current study aimed on determining the profile of parasuicide cases attending Brits Hospital, North West Province of South Africa, from 2013-2018.

Different methods, e.g. ingestion of a product, as well as injury, have been applied in parasuicide, ${ }^{3}$ referred to as an apparent attempted suicide without the actual intention of killing oneself. ${ }^{1,3}$ Parasuicide, as presented in the literature, has a prevalence that continues to increase every year. ${ }^{3}$ 
In Europe, the multicentre study on parasuicide conducted by the World Health Organization (WHO) focussed on two axes, one the epidemiologic monitoring of the problem and the second on the prediction of repetitive attempts at parasuicide. ${ }^{4}$ Data collected from 15 centres on the continent showed that the incidence of the phenomenon differs according to location as well as to gender. There were more males, 414 per 100,000 in Helsinki, capital of Finland while Leiden, in the Netherlands, had a lower total of 61 per 100,000. Statistics for women show that Pointoise, a town in France, had 595 versus 95 in Guipuzcoa, a municipality in Spain. Females are experiencing parasuicide to a greater degree than males do on the continent, with incidences respectively at 222 versus 167 . With regard to age, a high incidence rate was noted among people aged 15-34 years while a low rate was observed among those aged 55 and older. Looking at the second axis of the study, the incidence of repeated crises also differs according to location, although there were no significant differences between the two genders. ${ }^{4}$

The Edinburgh, United Kingdom study showed some trends regarding parasuicide rates. There was an increased rate among young, working-class people, especially for men, as well as women, with a history of alcoholism and psychotropic drug use. There was no change in other variables, e.g. repetition rates, use of non-prescribed drugs, etc. Hence the issue of changing trends in parasuicide remains questionable. $^{5}$

General practitioners (GPs) have a crucial role to play in the management of parasuicide patients in Australia. Thirty-eight percent of the Australians who present for parasuicide at the emergency units of public hospitals have consulted their GP during the previous week. Women younger than 35 years of age, with a history of parasuicide, constitute the major proportion of this group in emergency units. They consult a GP for somatic issues and a few days later present to a public hospital with a history of parasuicide. The Australian study revealed that only $2.3 \%$ of this age group had presented at the GP consultation with symptoms related to parasuicide. As a result, it is difficult for a GP to recognize the phenomenon at an early stage. These patients use medication prescribed by a GP to overdose themselves. The study analysis showed no significant differences between females and males or between young and older males. To minimize the problem in the entire region, GPs' training in parasuicide behaviours has to be given careful consideration. ${ }^{6}$

In an Islamic country such as Pakistan, three-and-a-half-year retrospective case reports revealed that young adults and married women were the most vulnerable. Marital conflicts appeared to be a weighty cause of stress for women in this country. Self-poisoning with benzodiazepines was the main method used to commit parasuicide in this part of the world. Parasuicide as an act is considered a criminal offence and condemned by the Islamic religion. ${ }^{7}$

A combined retrospective and prospective study was conducted in one of the Egyptian poisoning centres. A total of 244 self-poisoning participants were enrolled and subjected to clinical, toxicological and psychiatric assessment. The target was to identify the various signs that could lead to parasuicide, to determine the most common toxic agents used in the parasuicide act and to put in place measures and strategies that would prevent parasuicide, considered as non-fatal suicidal behaviour. Results indicated 
that adolescents (19 years), single, female, unemployed, with familial problems, exposed to emotional situations with a secondary or tertiary level of education were those most vulnerable to parasuicide. Looking at the seasons, more cases were registered during spring, followed by summer with winter last. The methods used were self-poisoning with a pesticide in $95 \%$ and drugs in $5 \% .{ }^{8}$

A quantitative study targeted 169 youths of parasuicide cases attended to in Chegutu, Zimbabwe. The youths were investigated in order to determine the risk as well as the protective factors associated with parasuicide in that area of the African continent. Analysis indicated that $39 \%$ of the youths presented with risk factors such as hopelessness, signs of illness, e.g. depression, alcohol and substance abuse, as well as stressful life events and family issues. Some protective factors were also revealed in association with the phenomenon of parasuicide in this study, i.e. religious beliefs, social support from peers and others. Further research was encouraged in order to cast more light on the question of risk versus protection regarding parasuicide. ${ }^{9}$

After applying the suicidal ideation questionnaire to 214 adolescents in the Western Cape province of South Africa, only participants with high scores were considered. Various risk factors associated with suicidal behaviour were identified, i.e. drug use, negative emotional experiences, lack of self-esteem, negative family atmosphere, conflict in family relationships, stressful life events, peer group pressure, romantic relationships and socioeconomic factors. ${ }^{10}$

While looking for the motives that led to parasuicide in Limpopo province, South Africa, a list comprising unemployment, poverty, domestic violence, mental health conditions including depression, other medical conditions such as HIV/AIDS, and accusation of witchcraft was established, following an unstructured indepth interview. From the association of these motives, an emotional state of living a meaningless life transpired. The suicidal ideation was gaining ground in the minds of nearly all respondents in this study. The methods used in different cases varied, based on knowledge and the determination to die. ${ }^{11}$

Nearly $10 \%$ of unnatural deaths among young people in South Africa are related to suicidal behaviour affecting almost all social demographic groups. This suicidal behaviour is increasing in prevalence and there are more suicide attempts with non-fatal outcomes than those with fatal outcomes. The ratios vary from 20:1 to 40:1. A study in Durban where these observations were made, showed that females, single, unemployed with low education, low-income level and part of the younger age group, were the most vulnerable when considering suicidal behaviour and, as a result, exposed to parasuicide. Depression seems to be a risk factor associated with suicidal behaviour. A screening strategy to diagnose depression may be considered in the comprehensive management of our patients, in order to prevent or reduce the parasuicide phenomenon. ${ }^{12}$

A one-year retrospective and descriptive study using patients' records was conducted in northern KwaZulu-Natal, South Africa. Only patients who presented at the casualty department of the hospital and had deliberate self-harm (DSH) as diagnosis qualified for this study because the target was to determine the profile of the patients and the reasons for DSH. The study results showed that the parasuicidal 
behaviour was affecting young, single, black women with a secondary level of education and those with relational problems more than other groups. Looking at the result (78\% parasuicide), a tool for the identification of clients at risk, as well as community intervention addressing the DSH issues, are needed in order to reduce the prevalence of this phenomenon in that area. ${ }^{13} \mathrm{~A}$ chart review of intentional selfpoisoning cases was retrospectively studied over a period of six months in Khayelitsha Hospital, Cape Town (South Africa). As a result, $68 \%$ of women with mean age of 27.3 years were affected; HIV infection was the comorbidity. The main method of this intentional self-poisoning was the ingestion of pharmaceuticals, e.g. paracetamol. Few benefited with $\mathrm{N}$-acetylcysteine (NAC) and benzodiazepines while $7.3 \%$ of the patients went to the high-level hospital and a death rate of $2 \%$ was registered. ${ }^{14}$

In a general hospital in South Africa, looking at the profile of 100 parasuicide patients referred to psychologists, the results showed that the most vulnerable were students and those who were unemployed, at rates of $79 \%$ and $16 \%$ respectively. The method used for parasuicide was ingestion of substances, e.g. paraffin, pesticides or battery acid. The precipitating factors were family conflicts, academic failure, teenage pregnancy, fear of Aids and mental illnesses. ${ }^{15}$

\section{Methods}

Aim

The current study aimed to determine the profile of parasuicide cases at Brits Hospital, North West Province of South Africa from 2013-2018.

\section{Characteristics of participants}

The study was based on a 5-year retrospective chart review of parasuicide cases attending Brits Hospital from 01 January 2013 to 01 January 2018. The setting of the study was Brits Hospital, a 200-bed hospital serving people in the vicinity of Madibeng (Brits), in the North West province of South Africa.

\section{Description of processes}

From the database, i.e. the electronic system of the Psychology unit of the hospital, the total number of parasuicide cases registered from 01 January 2013 to 01 January 2018 was 499 . All files were retrieved and assessed based on the required information for the current study; only 477 complied $(n=477)$. Convenience sampling was applied.

Each file was marked with a number, e.g. 1,2, 3, etc. in order to avoid capturing more than once, on the Excel spreadsheet, the information from the same file. The Excel spreadsheet was designed to ensure that all objectives of the study could be met. The design encompassed the socio-demographics, the methods used for parasuicide, period of the year the event took place, history of previous medical as well as psychiatric conditions of the patient, and the reasons motivating the patient to commit parasuicide.

\section{Type of statistical analysis used}


The study data was captured in an Excel spreadsheet, raw data was coded and data then imported into SPSS version 26 for statistical analysis. Pearson's chi-squared test was performed to compare association between categorical variables and determine the significance of their association with $p<$ 0.05. The results are presented in tables (frequencies and percentages included) and figures (graphs included).

\section{Results}

The age of the 477 participants ranged from 12-64 years (median $=24$ years), the mean age of the participants was 26.6 years (std. dev. $=9.4$ ), and the average was 21 years. The majority of participants $(257 ; 53.9 \%)$ were in the $16-25$ age range. A high number of cases was noted among females (297; $62.3 \%)$, Africans $(391 ; 82.8 \%)$, single $(352 ; 73.8 \%)$; unemployed $(304 ; 63.7 \%)$ and those with a secondary level of education $(300 ; 62.9 \%)$ as presented in Table 1 (see at the end of the article). 
Table 1

Sociodemographic characteristics of the study population $(n=477)$

\begin{tabular}{|c|c|c|}
\hline Characteristics & Frequency & Percentage (95\% Cl) \\
\hline Age (mean + std. dev.) & 26.6 year +9.4 & \\
\hline Age group & 14 & $2.94(1.76-4.87)$ \\
\hline $0-15$ & 257 & $53.88(49.39-58.30)$ \\
\hline $16-25$ & 131 & $27.46(23.65-31.64)$ \\
\hline $26-35$ & 51 & 10.69 (8.23-13.79) \\
\hline $36-45$ & 18 & $3.77(2.40-5.89)$ \\
\hline $46-55$ & 6 & $1.26(0.58-2.72)$ \\
\hline \multicolumn{3}{|l|}{$56-65$} \\
\hline Sex & 180 & $37.74(33.50-42.17)$ \\
\hline Male & 297 & $62.26(57.83-66.50)$ \\
\hline \multicolumn{3}{|l|}{ Female } \\
\hline Race & 391 & $82.84(79.18-85.97)$ \\
\hline African & 28 & $5.93(4.14-8.44)$ \\
\hline White & 38 & 8.05 (5.92-10.86) \\
\hline Coloured & 15 & $3.18(1.94-5.18)$ \\
\hline Asian & 5 & \\
\hline \multicolumn{3}{|l|}{ Missing } \\
\hline Marital status & 352 & $73.79(69.67-77.54)$ \\
\hline Single & 92 & $19.29(16.00-23.07)$ \\
\hline Married & 4 & $0.84(0.33-2.14)$ \\
\hline Widowed & 29 & $6.08(4.27-8.60)$ \\
\hline \multicolumn{3}{|l|}{ Divorced } \\
\hline Employment status & 73 & $15.30(12.35-18.81)$ \\
\hline Employed & 304 & $63.73(59.32-67.92)$ \\
\hline Unemployed & 98 & $20.55(17.16-24.40)$ \\
\hline Student & 2 & $0.42(0.12-1.52)$ \\
\hline Pensioners & & \\
\hline
\end{tabular}




\begin{tabular}{|lll|}
\hline Characteristics & Frequency & Percentage $(95 \% \mathrm{Cl})$ \\
\hline Level of education & 1 & $0.21(0.04-1.18)$ \\
None & 140 & $29.35(25.44-33.59)$ \\
Primary & 300 & $62.89(58.47-67.11)$ \\
Secondary & 36 & $7.55(5.50-10.27)$ \\
Tertiary & & \\
\hline
\end{tabular}

The largest number was observed in $2018(87 ; 18.24 \%)$, while the months of January and February had a high number of cases, respectively 65 (13.6) and 64 (13.4\%). The majority of cases represented a first attempt $(430 ; 90.2 \%)$. Overdose of medication $(300 ; 62.9 \%)$ was the most common method used. Many patients $(409 ; 85.7 \%)$ did not have a previous medical or psychiatric history. A relationship problem (207; $43.4 \%$ ) was the main reason for engaging in parasuicide as presented in Table 2 (see at the end of this article). 
Table 2

Description of parasuicide cases and associated past medical history $(n=477)$

\begin{tabular}{|lll|}
\hline Characteristics & Frequency & Percentage (95\% Cl) \\
\hline Year of occurrence & 85 & $17.82(14.65-21.51)$ \\
2013 & 68 & $14.26(11.40-17.68)$ \\
2015 & 86 & $18.03(14.84-21.73)$ \\
2016 & 75 & $15.72(12.73-19.26)$ \\
2017 & 87 & $18.24(15.03-21.95)$ \\
2018 & 76 & $15.93(12.92-19.49)$ \\
\hline Number of attempts & & \\
Once & 430 & $90.15(87.14-92.51)$ \\
Twice & 31 & $6.50(4.62-9.08)$ \\
Thrice & 14 & $2.94(1.76-4.87)$ \\
Four times & 2 & $0.42(0.12-1.52)$ \\
Method used to attempt parasuicide & 300 & $62.89(58.47-67.11)$ \\
Overdose & 6 & $1.26(0.58-2.72)$ \\
Paraffin ingestion & 64 & $13.42(10.65-16.77)$ \\
Detergent ingestion & 10 & $2.10(1.14-3.82)$ \\
Battery acid & 83 & $17.40(14.26-21.06)$ \\
Pesticide ingestion & 4 & $0.84(0.33-2.14)$ \\
Rape & & $2.10(1.14-3.82)$ \\
Combination(s) & 409 & $85.74(82.32-88.60)$ \\
History of medical or psychiatric illness & 68 & \\
Yes & & \\
No & & \\
\hline & & \\
\hline
\end{tabular}




\begin{tabular}{|lll|}
\hline Characteristics & Frequency & Percentage (95\% Cl) \\
\hline Reason for attempting parasuicide & 13 & $2.73(1.60-4.61)$ \\
Chronic illness & 11 & $2.31(1.29-4.08)$ \\
Academic failure & 207 & $43.40(39.02-47.88)$ \\
Relationship problem & 25 & $5.24(3.57-7.62)$ \\
Family problems & 140 & $29.35(25.44-33.59)$ \\
Unemployment & 8 & $1.68(0.85-3.27)$ \\
Personal problems & 35 & $7.34(5.32-10.03)$ \\
Orphaned & 4 & $0.84(0.33-2.14)$ \\
Substance abuse & 34 & $7.13(5.15-9.80)$ \\
Financial problems & & \\
\hline
\end{tabular}

More parasuicide cases were seen during the months of January and February, 65 and 64 cases respectively.

During the five years from 01 January 2013 to 01 January 2018, high rates of parasuicide cases were noted during the odd years and varied from 85-87 per month; low rates were seen during the even months and varied from 68-76. If taken separately, both groups showed an increase in numbers and a zig zag pattern from 2013 to 2018.

\section{Measure Of Association Between Variables}

In Table 3 only associations with statistical significance are presented. There is association between sex (male) and method used (battery acid; rape) with $p$-value 0.009 . Between age-group and reason (orphaned) the $p$-value was 0.000 ; reason and marital status ( $p$-value 0.000 ), number of attempts (twice or thrice) and marital status (married; widowed) with $p$-value 0.001 ; employment status (student and employed), and method used (pesticides and rape), as well as between employment status (student) and reason for parasuicide (relationship problem and orphaned). 
Table 3

Statistical significance noted in associations between variables

\begin{tabular}{|c|c|c|}
\hline First variable & Second variable & $\begin{array}{l}p- \\
\text { value }\end{array}$ \\
\hline Method used (battery acid; rape) & Sex & 0.009 \\
\hline Age group & Reason for parasuicide (orphaned) & 0.000 \\
\hline Reason for parasuicide & Marital status & 0.000 \\
\hline Number of attempts (twice; thrice) & Marital status (married; widowed) & 0.001 \\
\hline $\begin{array}{l}\text { Employment status (student and } \\
\text { employed) }\end{array}$ & Method used (pesticide and rape) & 0.002 \\
\hline Employment status (student) & $\begin{array}{l}\text { Reason for parasuicide (relationship problem; } \\
\text { orphaned) }\end{array}$ & 0.002 \\
\hline
\end{tabular}

\section{Discussion}

With respect to the socio-demographics in parasuicide, more specifically the age group vulnerability, the current study has found that young people from 16-25 years of age were vulnerable, but in adulthood the phenomenon decreased from that of the youth. In the multicentre European study ${ }^{4}$, people from 1534 years of age were more exposed and in adulthood, similar to the current study, fewer cases presented. ${ }^{4}$ Without limiting the age groups, the Edinburgh study in the United Kingdom ${ }^{5}$, in Pakistan ${ }^{7}$ as well as in Chegutu, or Zimbabwe ${ }^{9}$ considered young people as the target group in relation to parasuicide. Although in the Australian study more patients were under 35 years of age, there were no statistical differences between young and adult Australians. ${ }^{6}$ Similarities were noted in Egypt ${ }^{9}$ where the most affected were adolescents around 19 years of age. In South Africa, although the age groups are not delimited, quite a number of studies conducted on parasuicide have highlighted younger age groups as susceptible to suffer this phenomenon. ${ }^{12-15}$

With respect to gender, females (62.3\%) have been considered by the current study as more vulnerable than males. This difference has been noted in the European multicentre study ${ }^{4}$, the Pakistan study ${ }^{7}$, the Egyptian study ${ }^{8}$, in Durban ${ }^{12}$ as well as in Cape Town ${ }^{14}$ (both in South Africa), while in Edinburg (United Kingdom $)^{5}$ as well as in Australia ${ }^{6}$ there were no significant differences between the sexes.

With regard to ethnicity more African (82.8\%) than other ethnic groups, were exposed to parasuicide in this Brits study (North West province of South Africa); this correlates with observations made in northern KwaZulu-Natal, South Africa. ${ }^{13}$ In the current research, single people (73.8\%) were more vulnerable to parasuicide. This was also noted in northern KwaZulu-Natal. ${ }^{13}$ However, the contrary was noted in Pakistan where married women have been identified as vulnerable. ${ }^{7}$ 
Unemployment (63.7\%) was found to be associated with parasuicide in the current study as it was also noted in Egypt, ${ }^{9}$ as well as in South Africa, more specifically in Limpopo ${ }^{11}$ and Durban ${ }^{12}$. Although more victims of parasuicide were at the secondary level of education, $62.8 \%$ in the current study, in Egypt more participants were currently at or had completed the secondary or tertiary level of education. ${ }^{9}$

Overdose was the method used in many cases of parasuicide (63.9\%) in Brits Hospital; this was also observed in Australia ${ }^{6}$ (where GP medication was used), in Pakistan, ${ }^{7}$ in Egypt, ${ }^{8}$ as well as in Cape Town, South Africa. ${ }^{14,15}$ In the Limpopo province of South Africa, methods varied according to the knowledge possessed by patients (both male and female) as well as their determination to commit the act. ${ }^{11}$

No previous health conditions, e.g. medical or psychiatric symptoms were found in most of the Brits patients (85.7\%) prior to their admission to hospital. By contrast, in the UK study, young people had a history of alcoholism and psychotropic drug use before the crisis; and in Australia, many of the victims had somatic symptoms that made them consult a GP before they were examined at the hospital. In Egypt and Zimbabwe, most patients experienced depressive features before they exposed themselves to the crisis. ${ }^{8,9}$ In the Western Cape, South Africa, a few cases were attributed to self-esteem issues and negative emotional experiences. ${ }^{10}$ And continuing in South Africa, in Limpopo province as well as in Durban, depression and other conditions such as HIV were noted among the parasuicide cases. ${ }^{11,12}$

The main reasons for parasuicide deduced from research at Brits Hospital were a relationship problem (43.4\%) followed by unemployment (29.4\%). Patients alluded to a relationship problem, or romantic relationship, in the Western Cape, South Africa ${ }^{10}$ as well as in Pakistan where marital conflicts were causing stress to women. ${ }^{7}$

\section{Conclusion}

This study painted a clear picture of parasuicidal cases treated at Brits Hospital, being younger between $16-25$, mainly female, single, unemployed, the method used for parasuicide mostly overdose of medication and done mainly in the months of January and February. Furthermore, reasons for the parasuicidal attempt were relationship problem and unemployment. In most of the cases, it was the first attempt.

\section{Recommendation}

Considering the increased numbers of parasuicide cases noted in the current study, education seems to be the weapon that could be used in the Brits/Madibeng community. Education on parasuicide should be provided at the hospital, clinics, various recreational areas, on social media, as well as in secondary schools where it should be incorporated with the curriculum.

\section{Strengths}


From the Madibeng local municipality demographics, in 2016 Brits/Madibeng had a population of $536,110 .{ }^{16}$ High school pupils constituted $6.8 \%$ of the population, which is calculated to 364,455 . While applying the calculator.net formula for sample size calculation with confidence level $95 \%$ at confidence interval 5 , the sample size would be $381 .{ }^{17}$ By using a sample size of 477 , there was an oversampling that gives strength to this study. A limitation to the study relates to ethnicity. According to the medical records, a high number of cases were African, and a smaller number was noted from other racial groups. Hence the result cannot be generalised to the entire Brits/Madibeng community.

\section{Limitations}

High number of cases was among the African (according to the medical record) and a weak number was noted from other racial groups, therefore, the result cannot be generalised to the entire Brits/Madibeng community.

\section{Abbreviations}

DSH deliberate self-harm

NAC N-acetylcysteine

WHO World Health Organization

\section{Declarations}

\section{Acknowledgements}

To Mr Stevens Kgoebane, from Family Medicine/Sefako Makgatho Health Sciences University, for his contributions in the analysis.

Professional editing: Dr Jane Murray (freelance editor).

\section{Competing interests}

The authors have declared that no competing interest exist

\section{Authors' contributions}

TB conceptualised the project, contributed in protocol writing, in data collection, in data analysis and in article writing, KN produced the list of patients and the psychologic assessment of each cases. DKN contributed in protocol and article writing. AA contributed in protocol writing and data analysis. $\mathrm{HO}$ contributed in protocol writing. IG contributed in protocol writing and English editing. CNSS contributed in protocol writing.

\section{Funding information}


The research received no specific grant from any funding agency in the public, commercial or not-forprofit sectors.

\section{Data availability}

Data sharing is not applicable to this article as no new data was created or analysed in this study.

\section{Disclaimer}

The views and opinions expressed in this article are those of the authors and do not necessarily reflect the official policy or position of any affiliated agency of the authors.

\section{Ethical considerations}

Approval to conduct the research has been granted by the Brits Hospital authority and from the Sefako Makgatho Research Ethics Committee (SMUREC/M/23/2020:IR) from whom a clearance certificate was issued. Anonymity was observed from the data collection until the end of the research process, while written consent and confidentiality were not required since the researcher did not meet with the patients, but dealt solely with the medical records.

\section{References}

1. Welch SS. A review of the literature on the epidemiology of parasuicide in the general population. Psychiatr Serv. 2001;52(3):368-75.

2. Sukeri K. Common agents used in parasuicide in Buffalo city. SAJP. 2009:15(3):63-66.

3. Brooksbank DJ. Suicide and parasuicide in childhood and early adolescence. Br J Psychiatry. 1985;146(5):459-463. https://en.oxforddictionaries.com/definition/parasuicide. Accessed 19 May 2019.

4. Platt S, Bille-Brahe U, Kerkhof A, Bjerke T, Crepet P, De Leo D, Haring C, Lonngyist J, Michel K, et al. Parasuicide in Europe: the WHO/EURO multicentre study on parasuicide. I. Introduction and preliminary analysis for 1989. Acta Psychiatr Scand.1992; 85(2): 97-104.

5. Pfaff JJ, Acres J, Wilson M. The role of general practitioners in parasuicide: a Western Australia perspective. Arch Suicide Res.1999; 5(3): 207-214.

6. Khan M. Parasuicide in Pakistan: experience at a university hospital. Acta Psychiatr Scand. 1996; 93(4): 264-267.

7. Shreeda S, Tawfika N, Mohammeda N, Elmahdib M. Toxic agents used for parasuicide in Damietta Governorate, Egypt. Middle East Current Psychiatry. 2011; 18:11-17.

8. Chiwander R, Maseko M. Risk and protective factors of parasuicide among the youths of Chegutu urban district. 2016.

http://ir.msu.ac.zw:8080/xmlui/bitstream/handle/11408/2169/REJOICE\%20PARASUICIDE.pdf? sequence=1\&isAllowed=y Accessed 01 May 2019. 
9. Berg HVD, Tancread H, Low D. Adolescents' perceptions of stressors contributing to suicidal behaviour. Southern African Journal of Social Work and Social Development. 2014; 6(2). www.upjournals.co.za>SWPR>article>download Accessed 08/08/2020

10. Obida M, Clark C, Govender I. Reasons for parasuicide among patients admitted to Tshilidzini Hospital, Limpopo Province: a qualitative study. S Afr J Psych. 2013;19(4):222-5.

11. Naidoo S, Naidoo U, Naidoo A. Unmasking depression in persons attempting suicide. SAFP. 2015;57(2):83-87.

12. Ani JO, Ross AJ, Campbell LM. A review of patients presenting to accident and emergency department with deliberate self-harm, KwaZulu-Natal, South Africa. Afr J Prm Health Care Fam Med. 2017;9(1): a1234.

13. Hoving DJ, Hunter LD, Gerber RJ, Lategan HJ, Marks CJ. The burden of intentional self-poisoning on a district-level public hospital in Cape Town, South Africa. AfJEM. 2018;8(3):79-83.

14. Mhlongo T, Peltzer K. Parasuicide among youth in a general hospital in South Africa. Curationis. 1999: 72-76file:///C:/Users/T\%20Bongongo/Downloads/735-2169-1-SM.pdf Accessed 19 May 2019.

15. Brits/Madibeng population: http://municipalities.co.za > Madibeng. Accessed 19 July 2020.

16. Sample size calculator: https://www.surveysystem.com/sscalc.htm Accessed 19 July 2020.

\section{Figures}

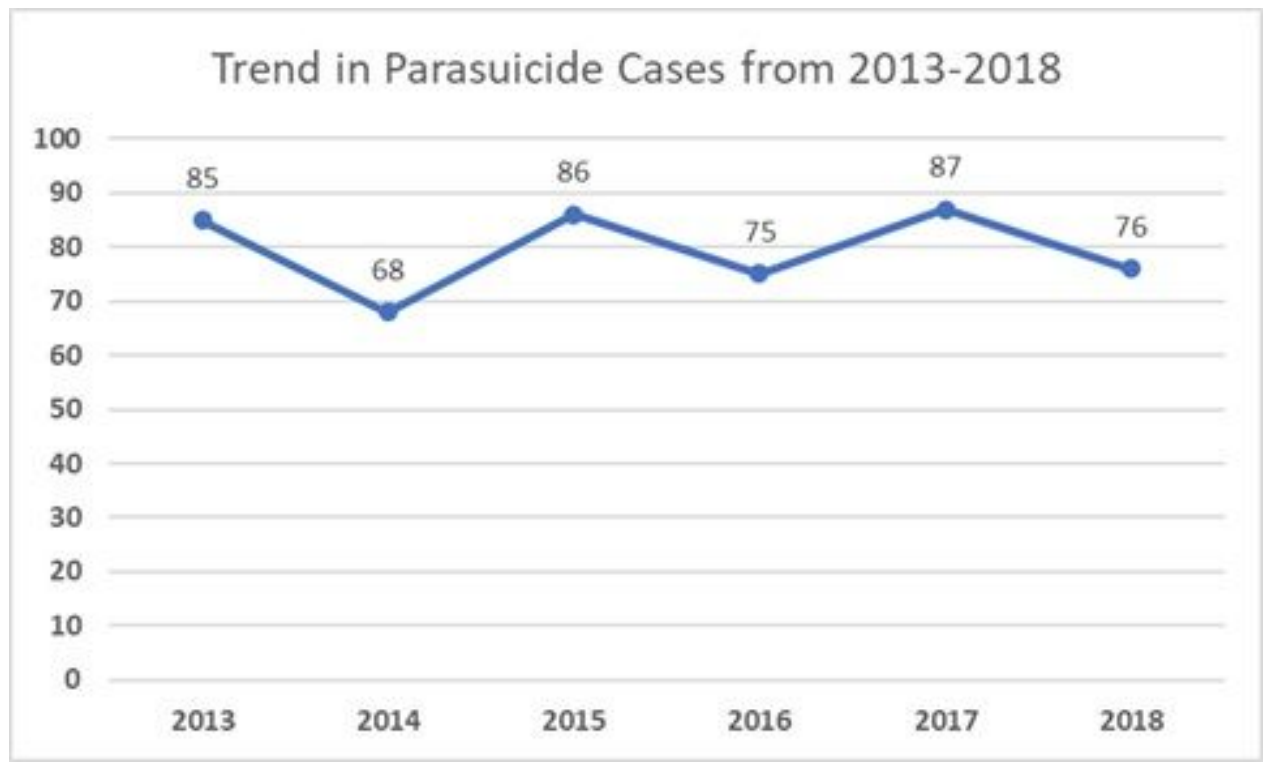

\section{Figure 1}

Trend in parasuicide cases from 2013 to 2018 
Monthly frequency of parasuicide cases: 2013-2018

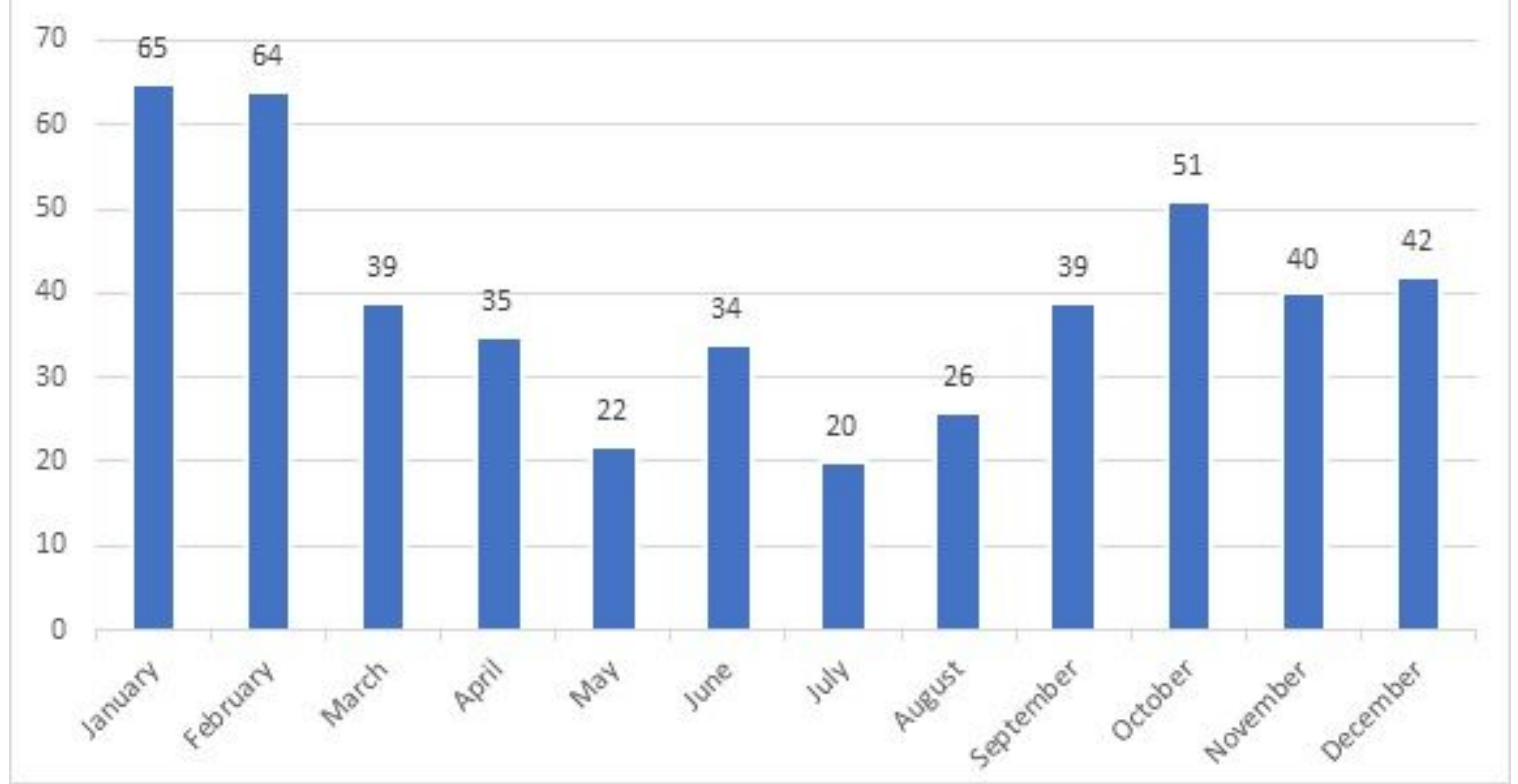

\section{Figure 2}

Monthly frequency of parasuicide cases at Brits Hospital: 2013-2018. More parasuicide cases were seen during the months of January and February, 65 and 64 cases respectively. 